\title{
How To Derive and Parameterize Effective Potentials in Colloid-Polymer Mixtures
}

\author{
P. G. Bolhuis*,t, and A. A. Louis ${ }^{\dagger}$ \\ Department of Chemistry, Lensfield Road, Cambridge CB2 1EW, UK, and Department of \\ Chemical Engineering, Nieuwe Achtergracht 166, 1018 WV, Amsterdam, The Netherlands \\ Received May 22, 2001; Revised Manuscript Received September 7, 2001
}

\begin{abstract}
Polymer chains in colloid-polymer mixtures can be coarse-grained by replacing them with single soft particles interacting via effective polymer-polymer and polymer-colloid pair potentials. Here we describe in detail how Ornstein-Zernike inversion techniques, originally developed for atomic and molecular fluids, can be generalized to complex fluids and used to derive effective potentials from computer simulations on a microscopic level. In particular, we consider polymer solutions for which we derive effective potentials between the centers of mass and also between mid-points or end-points from simulations of self-avoiding walk polymers. In addition, we derive effective potentials for polymers near a hard wall or a hard sphere. We emphasize the importance of including both structural and thermodynamic information (through sum rules) from the underlying simulations. In addition, we devel op a simple numerical scheme to optimize the parametrization of the density-dependent polymer-polymer, polymer-wall, and polymer-sphere potentials for dilute and semidilute polymer densities, thus opening up the possibility of performing large-scale simulations of colloid-polymer mixtures. The methods devel oped here should be applicable to a much wider range effective potentials in complex fluids.
\end{abstract}

\section{Introduction}

Binary mixtures of colloidal particles and nonadsorbing polymers have received renewed and growing attention recently, in part because they exhibit complex and interesting structure, phase behavior, interfacial properties, and rheology ${ }^{1-11}$ and in part because they are excellent model systems for the study of large length and time scale separations in complex fluids. Problems with bridging length scales are immediately apparent in even the simplest models of colloid-polymer mixtures: while the mesoscopic colloidal particles can be modeled as hard convex bodies, the polymers are often treated at the microscopic (Kuhn) segment level. Thus, even though the average size of the polymer coils may be of the same order of magnitude as that of the colloids, the number of degrees of freedom needed to model the former may be several orders of magnitude larger than what is needed for the latter. This naturally provokes the question: Can the polymers also be modeled as single composite particles? In fact, this is exactly what was done by Asakura and Oosawa (AO), who, in their classic work on colloid-polymer mixtures, ${ }^{12,13}$ modeled the polymers as ideal particles with respect to each other and as hard spheres with respect to the colloids. This model is strictly speaking only valid for noninteracting polymers or for interacting polymers in the dilute limit, while many interesting phenomena such as polymer-induced phase separation take place at finite concentrations of interacting polymers. Our ultimate goal, therefore, is to go well beyond the AO model and describe nonideal polymers in a good solvent up to semidilute densities. We recently extended the AO concept to take into account polymer-polymer interactions, first by rather naively adding a Gaussian repulsion between the polymers ${ }^{14}$ and then by carrying out a much more sophisticated program which resulted in

\footnotetext{
† Department of Chemistry.

₹ Department of Chemical Engineering.
}

density-dependent polymer-polymer and polymer-wall pair interactions ${ }^{15,16}$ or, in a complementary approach, density-independent many-body interactions. ${ }^{17}$

In this paper we revisit the route to the densitydependent pair potentials in much more detail than in our previous work. ${ }^{15,16}$ These density-dependent interactions are derived by inverting structural information, namely the center-of-mass (CM) radial distribution function $g(r)$ for the polymer-polymer interactions and the wall-polymer or sphere-polymer CM density profile $\rho(r)$ for the polymer-wall or polymer-sphere interactions, respectively. We focus here on the considerable subtleties inherent in these inversions, in particular the importance of using thermodynamic information or related sum rules to achi eve accurate potentials. Many of the lessons learned should be valid for a wider set of effective potentials. As a first example, we derive effective potentials for polymer solutions based on midpoint and end-point representations.

We also revisit the problem of deriving effective potentials for a wall-polymer interaction, again emphasizing the subtleties involved in the inversion process. In addition, we show how to use a method similar to derive accurate sphere-polymer potentials, a key step toward large-scale simulations of colloid-polymer mixtures. Whereas the integral over the potentials holds the key to accurate thermodynamics in the homogeneous polymer case, here the relative adsorption, defined as the integral over the density profile near a wall or sphere, is the key to achieving the correct thermodynamics.

We also derive a parametrization scheme for the potentials used in the homogeneous and the inhomogeneous systems. To achieve this, we use a novel Monte Carlo scheme which should be easily adaptable to a wider class of effective potentials.

The paper is organized as follows: In section 2 we repeat the most important aspects of the models and simulation methods we used. In section 3 we describe 
how to derive polymer-polymer, polymer-wall, and polymer-sphere effective potentials from self-avoi ding walk (SAW) polymer simulations and discuss the subtle ties involved in the inversion procedures. We also derive other representations of the polymer-polymer potential, such as the mid-point or end-point representation. Section 4 contains the fitting procedures we use to parametrize the density-dependent potentials. We end with the customary concluding remarks.

\section{Simulation Models and Methods}

Simple lattice models, such as the self-avoiding walk (SAW), are widely used to describe flexible, uncharged polymers in a good solvent. Their popularity stems in part from the fact that, even though they ignore all chemical details except excluded volume and polymer connectivity, these models can still reproduce the scaling behavior and many other physical properties of athermal polymer solutions. ${ }^{21,22}$ In addition, because of their simplicity, these models are ideally suited for highly efficient numerical simulation algorithms. The SAW model consists of a cubic lattice of size $\mathrm{M}$ on which $\mathrm{N}$ chains of $\mathrm{L}$ monomers are placed. Repulsion between (monomer) segments is built in by allowing only one segment per lattice site. The monomer concentration is given by $c=N L / M$, while the polymer chain concentration is given by $\rho=\mathrm{N} / \mathrm{M}$. The size of the polymers is determined from the radius of gyration $R_{g}$, which, for an isolated chain, scales as $\mathrm{R}_{\mathrm{g}} \sim \mathrm{L}^{0.59}$.

Solutions of flexible polymers in a good solvent can be classified as a function of polymer concentration into the dilute, semidilute, and melt regimes, each with different scaling behavior. The density or concentration at which, roughly speaking, the dilute regime crosses over to the semidilute regime is called the overlap concentration or density $\rho^{*}$, defined as ${ }^{4} / 3 \pi \rho^{*} \mathrm{R}_{\mathrm{g}}{ }^{3}=1$, and corresponding to 1 polymer per unit volume $4 / 3 \pi R_{g}{ }^{3}$. In both these regimes, the monomer concentration $c \ll$ 1 , and the only rel evant length scale is $R_{g}$. When, upon increasing the polymer density, the monomer concentration becomes appreciable, the system crosses over to the so-called melt regime where the monomer size becomes an additional relevant length scale. Thus, when modeling the semidilute regime, it is important to take polymer chains that are long enough, to ensure that $c$ is still small. The monomer density $\mathrm{c}^{*}$ at the overlap concentration $\rho^{*}$ scales roughly as $\mathrm{C}^{*} \approx 4 \mathrm{~L}^{-0.8}$ for SAW lattice chains (see ref 16), which implies that for polymers of finite length, there is only a limited semidilute regime. We studied polymers with lengths $\mathrm{L}=$ 500 and $\mathrm{L}=2000$. For an isolated $\mathrm{L}=500$ coil the radius of gyration 40 is $R_{\mathrm{g}}=16.5 \pm 0.02$ so that $\mathrm{c}=0.027$ at $\rho=\rho^{*}$, and $\mathrm{c}=0.23$ at $\rho / \rho^{*}=8.70$, the highest density we study for this length. For $\mathrm{L}=2000$ chains, $\mathrm{R}_{\mathrm{g}}=$ $37.45 \pm 0.04$, so that $\mathrm{c}=0.009$ at $\rho=\rho^{*}$, and $\mathrm{c}=0.064$ at $\rho / \rho^{*}=7.04$, the highest density studied for $\mathrm{L}=2000$ polymers. This suggests that while we do not expect the $\mathrm{L}=2000$ polymers to exhibit any significant corrections to scaling behavior for the densities studied, the $\mathrm{L}=$ 500 polymers may begin to deviate slightly from the true semidilute regime at the highest densities. We established previously that the second virial coefficient for two $\mathrm{L}=500$ polymers is close to the scal ing limit. ${ }^{16}$ This indicates that for low densities the effective potentials, obtained by the coarse graining procedure, are very near the scaling limit where properties only depend on $R_{g}$ and not on $\mathrm{L}$.
The SAW simulations were performed by employing the Monte Carlo pivot algorithm ${ }^{23,24}$ together with translational moves. This simple algorithm is sufficient to sample the configuration space at low concentration. In the semi di lute regime, i.e., for concentrations $\rho>\rho^{*}$, we use configurational bias M onte Carlo algorithms. ${ }^{25,26}$

\section{Deriving Effective Pair Potentials}

The central theme of the coarse graining procedure advocated here and in previous work ${ }^{15,16}$ is to replace each polymer by a single particle, interacting with the other polymer particles via an effective (pair) potential. For complex particles like polymers, there is some freedom in the choice of coordinate for the single "particle". We have mainly used the CM, but one could also use the average monomer, the end-points, or the mid-point as a monomer-based representation (see for example the appendix of ref 16 or 27). We therefore discuss the mid-point and end-point representations in section 3.2; a more complete analysis is planned for a future publication. ${ }^{28}$

3.1. CM-CM Potentials for Homogeneous Polymer Solutions. In the $\rho \rightarrow 0$, or low-density, limit, the effective potentials can be derived from the logarithm of probability of overlap of the CM of two polymer coils. ${ }^{10,17}$ Calculating this overlap probability involves integrating over the polymer degrees of freedom by the Monte Carlo procedures described in section 2. We sample the configurations of two polymers an infinite distance apart with the pivot algorithm, and after every 1000 pi vot moves we accumulate an overlap probability histogram by testing for segment overlap as a function of the CM distance. The effective potential determined in this manner is approximately Gaussian in shape, with a range of the order $\mathrm{R}_{\mathrm{g}}$ for all lengths. When the CM's completely overlap, the potential has a maximum of $v(r=0)=1.88 \pm 0.01$ for the $L=500$ polymers and $\mathrm{v}(\mathrm{r}=0)=1.82 \pm 0.02$ for the $\mathrm{L}=2000$ polymers, which is very close to the scaling limit estimate $v(r=0)=1.80$ \pm 0.05 . $^{16}$ (Since all interactions are of entropic origin, we set $\beta=1 / \mathrm{k}_{\mathrm{B}} \mathrm{T}=1$ throughout this paper.) In the scaling limit the potentials depend only on $R_{g}$, so that the probability for complete overlap of the CM's of two polymers will be independent of their length $L$.

At finite densities there is no longer a simple logarithmic relationship between overlap probabilities and the effective potentials. ${ }^{10,17}$ Instead, we use the one-toone mapping ${ }^{19,20}$ between $g(r)$ and $v(r)$ to find, for each density, the unique effective potential that exactly reproduces the two-body correlations. To generate the pair correlations, we performed MC simulations of $\mathrm{L}=500$ polymers on a $240 \times 240 \times 240$ cubic lattice, for several different number of polymers ranging from $\mathrm{N}=50\left(\rho / \rho^{*}=0.07\right)$ to $\mathrm{N}=6400\left(\rho / \rho^{*}=8.70\right)$. F or $\mathrm{L}=$ 2000 polymers we used a lattice of $500 \times 500 \times 500$ units, and the number of polymers ranged from $\mathrm{N}=$ $200\left(\rho / \rho^{*}=0.35\right)$ to $\mathrm{N}=4000\left(\rho / \rho^{*}=7.04\right)$. During the simulations we collected the radial distribution functions between the CM of the polymers. We typically needed on the order of $10^{7} \mathrm{M}$ onte Carlo moves to achieve sufficient accuracy.

The effective potentials $v(r ; \rho)$ were constructed using the one-component OZ equation, supplemented by the hypernetted chain closure, ${ }^{29}$ which is nearly exact for the type of potentials we generate. ${ }^{30}$ The resulting effective pair interactions are plotted in Figure 1 and Figure 2 for the $L=500$ and $L=2000$ polymers, 


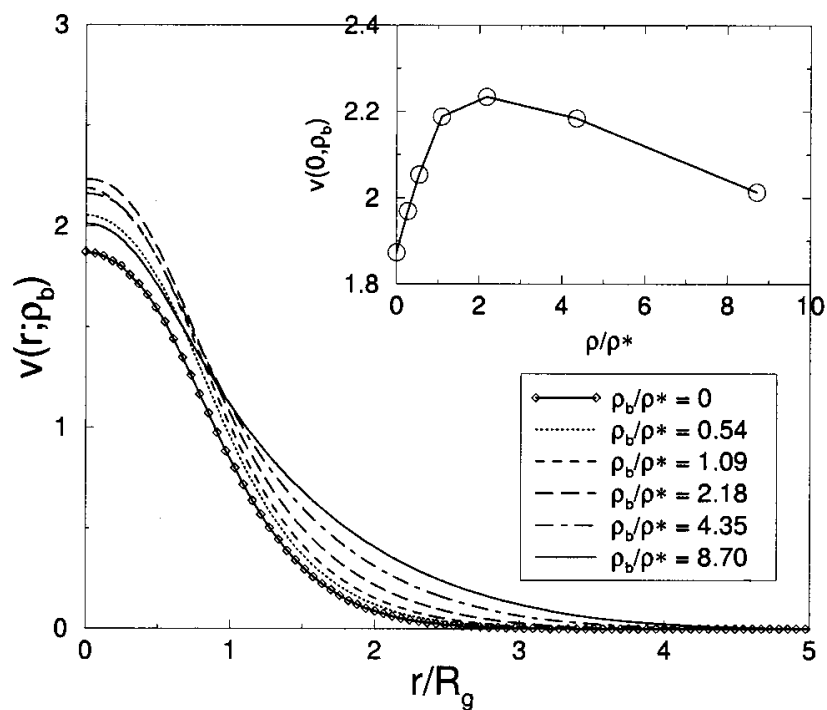

Figure 1. Effective polymer $C M$ pair potential $v(r ; \rho)$ for $L=$ 500 derived from an OZ-HNC inversion of $g(r)$ for different densities. The $x$-axis denotes $r / R_{g}$, where $R_{g}$ is the radius of gyration of an isolated SAW polymer. Inset: the value of the effective polymer CM pair potential at $r=0$ as a function of density $\rho / \rho *$. The maximum of the potential initially increases before decreasing at high concentration. For clarity, we left out the lowest densities.

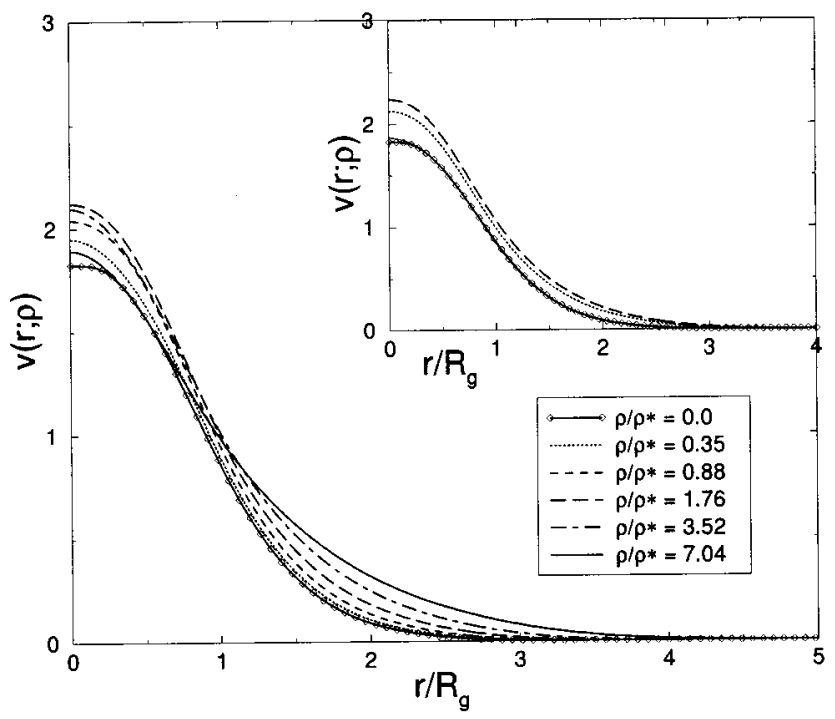

Figure 2. Effective polymer $C M$ pair potential $v(r ; \rho)$ for $L=$ 2000. Inset: a comparison with Figure 1 for $\rho / \rho^{*}=0$ (solid lines) shows that we are very near the scaling limit. This is similar for finite density, e.g., the effective potential for $\rho / \rho^{*}$ $=1.76 ; \mathrm{L}=2000$ (dotted line) is very close to the $\rho / \rho^{*}=2.18$, $\mathrm{L}=500$ potential (dashed line).

respectively, and show a clear though surprisingly small density dependence. This density dependence can be understood from the effect of the density-independent many-body interactions ${ }^{17}$ which, for $\rho / \rho^{*} \leq 1$, are dominated by the three-body interactions.

The basic principles of the inversion procedure were al ready described in ref 16 , but here we want to point out some important subtleties that must be kept in mind when performing such inversions.

(1) First of all, it is important to generate accurate $g(r)$ 's. This is illustrated in Figure 3, where two different potentials from Figure 1, namely the potential at $\rho=0$ and the potential at $\rho / \rho^{*}=1.09$, were used at the same density $\rho / \rho^{*}=1.09$ to generate the radial distribution

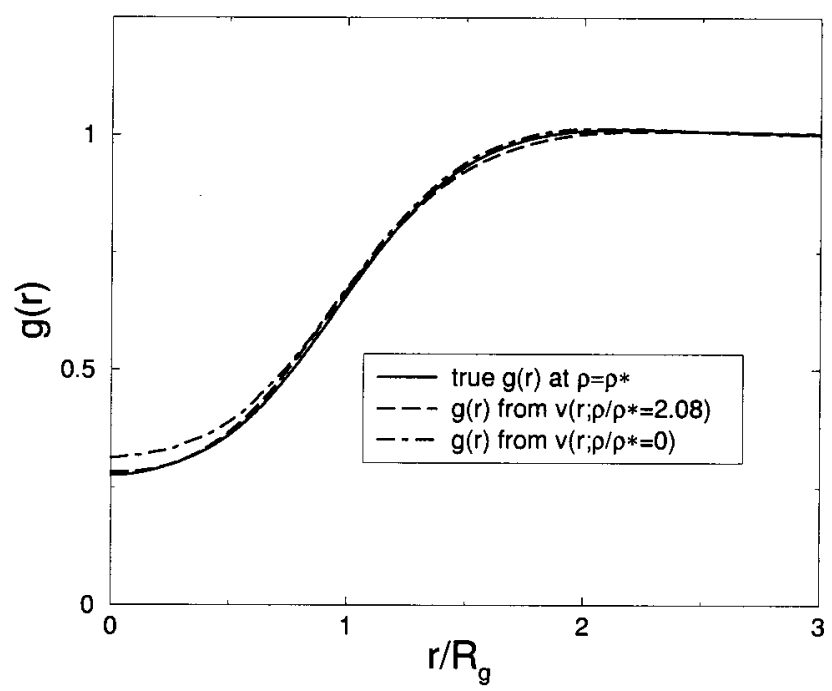

Figure 3. Comparison of $g(r)$ 's generated at density $\rho=\rho^{*}$ by the low-density potential $v(r ; \rho=0)$ and the higher density potential $v\left(r ; \rho / \rho^{*}=2.18\right)$ compared to the true $g(r)$ at that density. Note how small the differences are.

function $g(r)$. These $g(r)$ 's are then compared to the true SAW $g(r)$ at that density. Even though the potentials seem quite different (see Figure 1), the differences in the radial distribution functions are small, implying that the process which generates the $g(r)$ must be significantly more accurate than the difference between the radial distribution functions in Figure 3 if one is to obtain accurate effective potentials.

(2) Second, because polymers do not have a hard core, one can achieve much higher number densities than is normally found for simple fluids. ${ }^{18,19}$ This also puts extra demands on the accuracy of the correlation functions, since at high densities very small errors tend to destabilize the $\mathrm{OZ}$ inversion procedure, making convergence difficult to achieve.

(3) Third, $g(r)$ is only known up to half the simulation box size, rendering the inversion problem underdetermined. If the potential is known for all $r$, OZ techniques can be used to extend $g(r)$ beyond the box size, ${ }^{31}$ but when one is trying to find $g(r)$ from simulations with an unknown $v(r)$, some assumption for the large $r$ behavior of $v(r)$ must be made. We assume that $v(r, \rho)$ $=0$ beyond half the box size, but this is not necessarily obvious at high density, and one needs large simulation boxes to achieve proper convergence of the effective potentials. We used boxes with sides of approximately $14 \mathrm{R}_{\mathrm{g}}$. Although it may seem that the potentials have vanished al ready at shorter distances, this is still near the minimum length necessary to ensure that there are no cutoff effects in the potentials, especially for higher densities. Note that our new Monte Carlo fitting procedure, described in section 4, partially hel ps overcome this problem.

An effective potential that correctly reproduces paircorrel ations should, in princi ple, also predict the correct thermodynamics through the compressibility equation. ${ }^{29}$ But we stress that correctly determining the equation of state (EOS) $Z=\Pi / \rho$ (here $\Pi$ is the polymer osmotic pressure) or other thermodynamic properties from effective potentials can also be quite subtle. We illustrate this in Figure 4 where we compare a typical potential and its fit to a single Gaussian. Although these potentials do not seem that different, and generate almost identical radial distribution functions $g(r)$, they result 


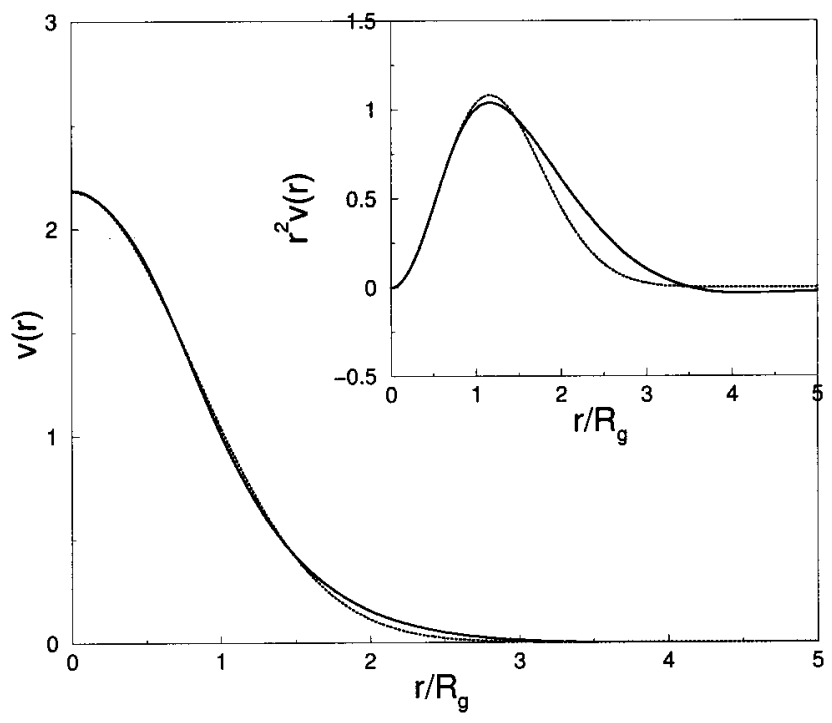

Figure 4. Comparison between the effective potential $v\left(r ; \rho=\rho^{*}\right)$ (solid line) and its fit to a single Gaussian (dotted line). Although the fit may appear to be quite good to the eye, the pressures obtained from these potentials differ by about $10 \%$. The reason for this is illustrated in the inset where the potential is multiplied by $r^{2}$, and the differences become more visible.

in pressures which differ by about $10 \%$. The origin of this difference is clear from the inset, where we plot $r^{2} v(r ; \rho)$, which is in fact a better measure of the rel evant differences between the potentials than $v(r ; \rho)$ itself. That $r^{2} v(r ; \rho)$ is a better indicator for the accuracy of the predicted pressure follows from the fact that potentials such as those shown in Figure 1, 2, or 4 lead to meanfield fluids, 30,32 so named because their equation of state approximately follows the simple mean-field form:

$$
\mathrm{Z} \equiv \mathrm{P} / \rho \approx 1+\frac{1}{2} \hat{\mathrm{v}}(0 ; \rho) \rho
$$

for a wide range of densities. Here, $\hat{v}(0 ; \rho)$ is the $k=0$ component of the Fourier transform of the pair interaction, which for spherical symmetric functions involves the integral over $r^{2} v(r ; \rho)$.

Keeping these subtleties in mind, we found very good agreement between the EOS generated by applying the compressibility equation to the effective potentials in Figures 1 and 2 and the EOS of the underlying SAW polymer solutions. The compressibility was calculated with the accurate HNC equation. The $L=2000$ results almost exactly fall onto the $L=2000$ EOS computed directly by the method of Dickman. ${ }^{33} \mathrm{~F}$ or $\rho / \rho^{*}<5$, the $\mathrm{L}=500 \mathrm{EOS}$ calculated with the effective potentials is very close to the directly computed one, but at higher densities some small differences devel op. We attribute these to the difficulties in achieving high accuracy for the tails of the potentials when the inversions are performed at these higher densities. We expect the $L=$ 500 EOS to be slightly higher than the $\mathrm{L}=2000$ one at higher $\rho / \rho^{*}$ because the monomer density c is higher for the shorter polymers, which induces L-dependent corrections to the scaling limit at the higher densities. Both the $\mathrm{L}=500$ and the $\mathrm{L}=2000 \mathrm{EOS}$, where $\mathrm{c}$ remains very small at the densities probed, are slightly higher than the EOS derived by renormalization group (RG) calculations. ${ }^{34,22}$ They approximately fol low the correct $\Pi / \rho \sim \rho^{1.3}$ scaling expected for the semidilute regime ${ }^{21}$ up to the highest densities. In contrast, if one were to

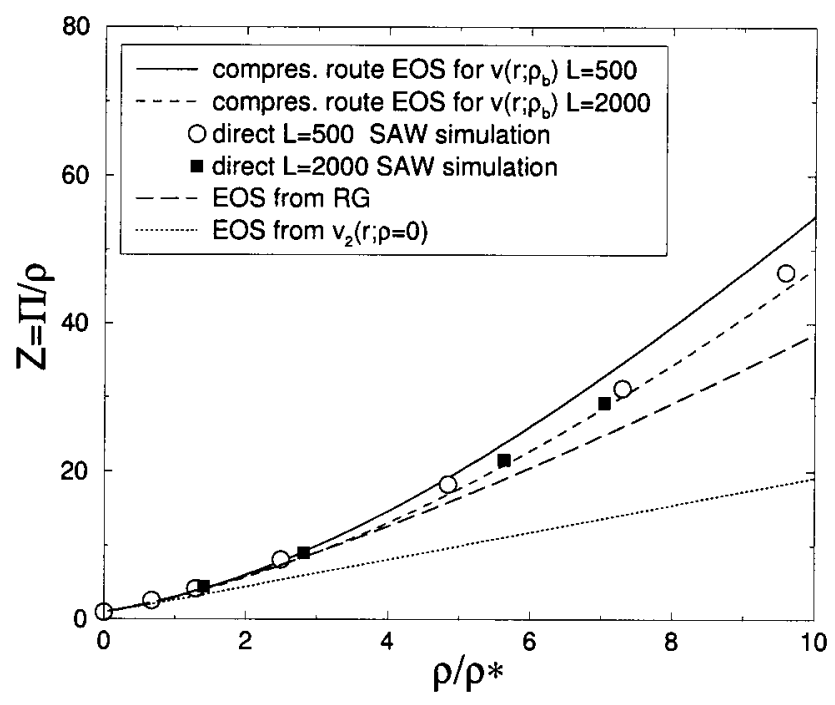

Figure 5. Linear plot of the $E O S Z=\Pi / \rho$ for $L=500$ and $L$ $=2000$ polymers, here determined from the effective potentials through the compressibility route and by direct simulations of the underlying SAW polymer system. The slight deviations for the $L=500$ case at the higher densities are most likely due to small inaccuracies in the inversion procedure used to generate the effective potentials. We also show the EOS derived from an $\mathrm{RG}^{34}$ approach. Using only the $\mathrm{Iim}_{\rho \rightarrow 0}$ form of $\mathrm{v}\left(r ; \rho / \rho^{*}\right)$ strongly underestimates the EOS.

use the $\mathrm{v}(\mathrm{r} ; \rho=0)$ potential at all densities, the EOS would be underestimated and would follow mean-field fluid behavior with the incorrect $\Pi / \rho \sim \rho$ scaling at large $\rho$ instead. So, even though Figure 3 shows that the $\rho=$ 0 potential results in pair correlations $g_{2}(r)$ that are similar to the true $g_{2}(r)$ 's, the effective thermodynamics can differ significantly. Since the density dependence of the effective pair potentials was shown to arise from the many-body interactions, ${ }^{17}$ this immediately suggests that the corrections to the simple linear $\rho$ scaling of the EOS are due to the three- and higher-body interactions.

3.2. Other Representations of Potentials for Homogeneous Polymer Solutions. As mentioned at the beginning of this section, one could also use other representations for effective pair potentials between polymers. For star polymers, for example, the mid-point is a more natural coordinate. ${ }^{36}$ The $\mathrm{f}=2 \mathrm{arm}$ limit of a star polymer would correspond to a normal linear polymer, but in the mid-point representation.

In Figure 6 we compare the mid-point, end-point, and CM representations of the interaction between two isolated polymer coils, all calculated in the usual way by taking the logarithm of the overlap probability for $L$ $=500$ SAW polymers on a lattice. We also compare the $\mathrm{f}=2 \mathrm{arm}$ limit of the star polymer interaction derived by Likos et al. ${ }^{36}$ as well as an improvement, especially tailored for linear polymers. ${ }^{37}$ The mid-point and endpoint representations have a divergence at full overlap that scal es as $\lim _{r \rightarrow 0} v(r) \propto \ln \left(r / R_{g}\right)$, so that they appear to be quite different from the CM representation. However, because the divergence is integrable in 3 dimensions, these potentials still result in mean-field fluids. ${ }^{10}$ This is illustrated in Figure 7 where we plot $4 \pi r^{2} v(r)$. The divergence has disappeared, and we see once again that it is the tails of the potentials, and not the small $r$ behavior, which matters for the thermodynamics. While in Figure 6 the differences between the two-arm limit of the star polymer potential and the true mid-point potential are quite small (just fractions of $k_{B} T$ ), Figure 7 , together with eq 1 , shows that they will 


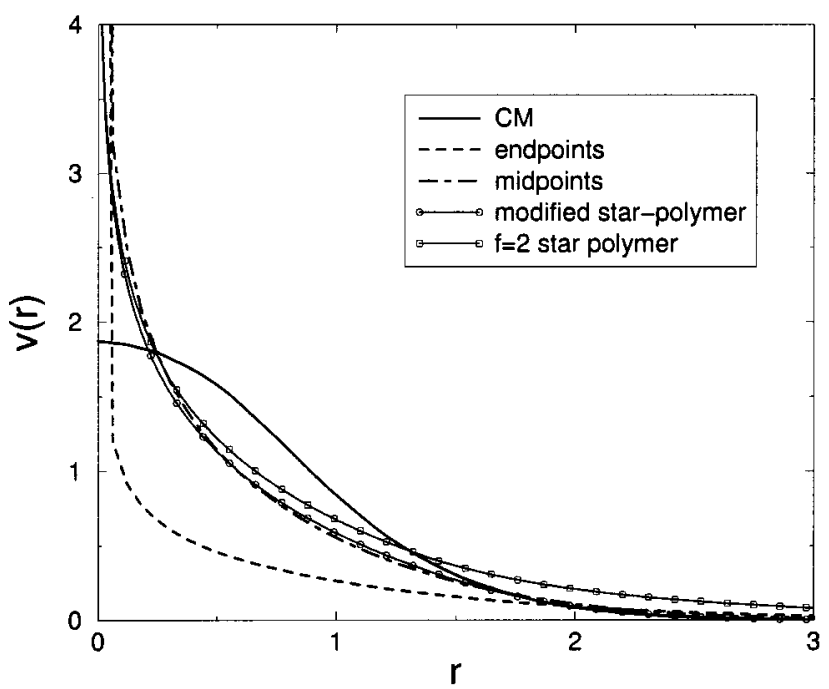

Figure 6. End-point, mid-point, and CM representations of the interaction $v(r)$ between two isolated polymers. Also included are two fits to the mid-point potentials taken from refs 36 and 37, as explained in the text.

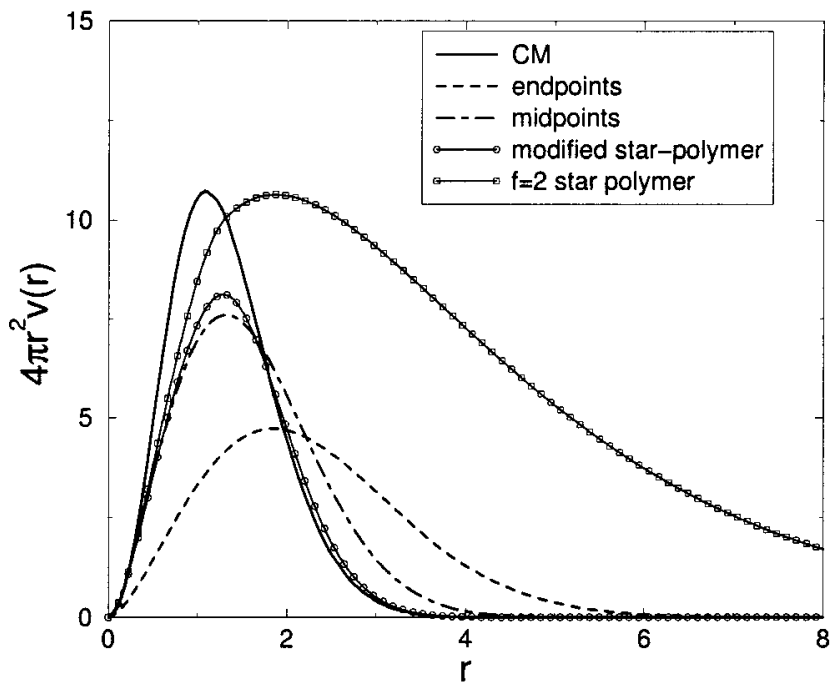

Figure 7. End-point, mid-point, and CM representations of the interaction $4 \pi r^{2} v(r)$ between two isolated polymers. Plotting the potentials in this way accentuates the differences. Note in particular how poor the $\mathrm{f}=2$ limit of the star polymer potential ${ }^{36}$ performs.

generate dramatically different EOS. But even the specially tail ored mid-point potential, which appears to almost perfectly follow the simulations in Figure 6, differs visibly when plotted as $r^{2} v(r)$. In contrast, for the SAW simulation, the three different representations, mid-point, end-point, and CM, should all lead to the sameEOS, provided that, as expected, the volume terms are similar and small.10,16

These observations demonstrate a more general point, al so mentioned in the previous section: J ust because a fit to a potential appears to be very close to the original potential does not necessarily mean it will generate the correct thermodynamic behavior; great care must be taken to ensure that the fit conserves the right quantities. (Note that the fit in ref 37 was indeed constrained to give the correct second virial coefficient, which will result in the correct low density thermodynamics.)

In principle, one can also derive density-dependent potentials for the mid-point or end-point representations, just as was done for the CM representation. In

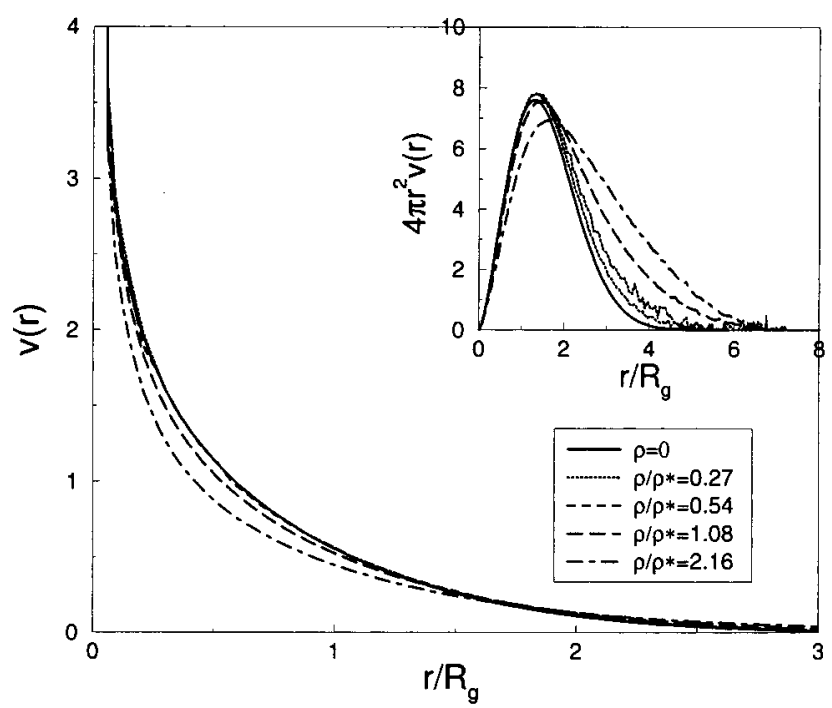

Figure 8. Mid-point $v(r)$ that exactly reproduces the midpoint-mid-point $g(r)$ for different bulk polymer densities. Inset: $4 \pi r^{2} v(r)$ shows that the more significant change is in the tails of the potentials.

Figure 8, we show the mid-point potentials which re produce the mid-point $\mathrm{g}(\mathrm{r})$ 's generated from direct SAW polymer simulations. We used the same HNC inversion techniques used earlier for the CM representation and expect similar accuracy. In summary then, while there is some flexibility in the choice of coordinates, many of the lessons learned for the CM representation carry right through to the other representations. ${ }^{28}$

3.3. Colloid-Polymer Potentials from Wall-OZ and Sphere-OZ Inversions. So far, we have only considered effective polymer-polymer pair potentials, but a full coarse grained description of a colloidpolymer mixture, or, more generally, of polymers in confined geometry, requires effective potentials between polymers and (colloidal) surfaces as well. We focus here on nonadsorbing surfaces and calculate the effective interaction between polymers and a hard wall and between polymers and a hard sphere (HS). The former is important for such systems as mixtures of polymers and platelets or mixtures of polymers and very large spheres; the latter helps provide a model for mixtures of spherical colloids and larger polymers.

Near a hard nonadsorbing surface, entropic effects create a polymer depletion layer, even if the chains themselves are noninteracting. Although there is no polymer-polymer interaction when modeling such ideal chains, there will still bea finite wall-polymer potential $\phi(\mathrm{z})$. This interaction can be found for ideal polymers near a wall by the simple result $\phi(\mathrm{z})=-\ln (\rho(\mathrm{z}) / \rho)$, where $\rho(z)$ is the polymer density at a distance $z$ from the surface and $\rho$ is the uniform bulk density. For polymers in an end-point representation, the interaction reduces to $\phi(z)=-\ln \left(e r f\left(z / 2 R_{g}\right)\right)$, while for polymers in the midpoint representation $\phi(z)=-2 \ln \left(\operatorname{erf}\left(z / \sqrt{2} R_{g}\right)\right) .{ }^{16} A$ similar result should follow in the CM representation, but we have not yet succeeded in obtaining an analytic form.

Asakura and Oosawa ${ }^{12,13}$ modeled polymers as ideal with respect to each other and with a hard-sphere potential $\phi(z)$ of range $R_{g}$ with respect to spheres or walls. Here we extend their work and calculate the $\phi(\mathrm{z})$ that exactly reproduces the density profiles $\rho(\mathrm{z})$ measured by direct simulations of SAW polymers near walls or spheres. 


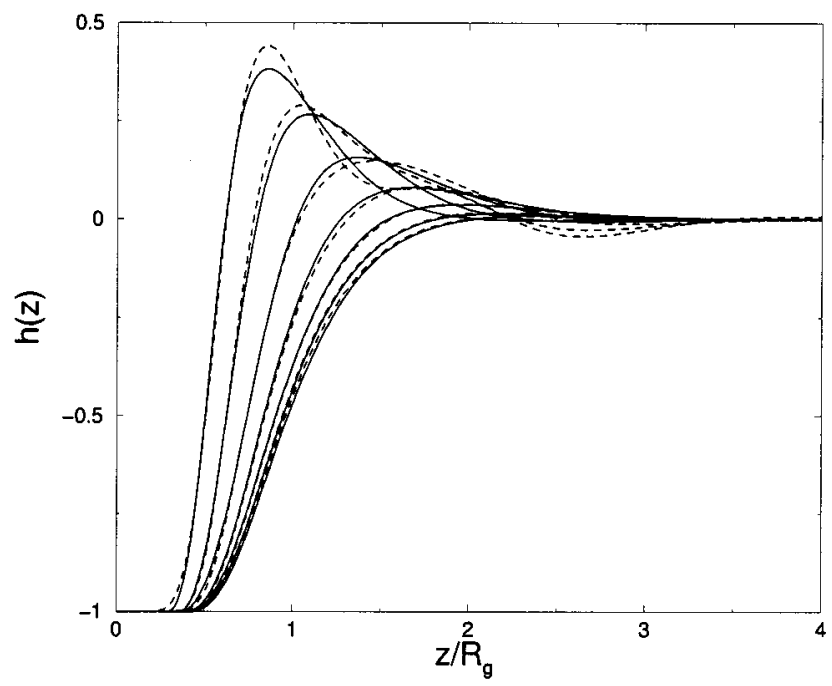

Figure 9. Measured $h(z)$ (solid lines) next to a wall obtained from SAW simulation compared with the $h_{f}(z)$ which follows from the optimized fitted potential $\phi_{\mathrm{f}}(z ; \rho)$ (dashed lines). From left to right the curves correspond to bulk polymer densities $\rho / \rho^{*}=2.27,1.16,0.59,0.30,0.16,0.08$, and 0 . Note that the densities differ slightly from the corresponding bulk density due to the depletion at the wall.

First we consider SAW polymers near a hard wall. The effective polymer-wall potentials $\phi(z ; \rho)$ can be derived from the $\rho(z)$ by combining the wall-OrnsteinZernike equation with the HNC closure, leading to

$$
\phi(\mathbf{z} ; \rho)=-\ln (\rho(\mathbf{z}) / \rho)+\rho \int \mathrm{d} \mathbf{r}^{\prime}\left(\rho\left(z^{\prime}\right) / \rho-1\right) c_{\mathrm{b}}\left(\left|\mathbf{r}-\mathbf{r}^{\prime}\right|\right)
$$

Here, $c_{b}(r)$ is the bulk polymer direct correlation function. The details of this inversion procedure are given in ref 16 . In contrast to the homogeneous case, the inversion involves only one iteration, because the bulk polymer-polymer potentials and hence $c_{b}(r)$ are al ready known.

Using the same explicit SAW polymer model as in section 2, we performed MC simulations of polymers of length $\mathrm{L}=500$ on a lattice of size $\mathrm{M}=240 \times 240 \times$ 240 with hard planar walls at $x=0$ and $x=240$. The polymer segments were not allowed to penetrate the walls. The simulations were done for $\mathrm{N}=0,100,200$, $400,800,1600$, and 3200. During each simulation, we computed the density profiles $\rho(z)$, where $z$ denotes the distance between the polymer CM and the wall. The normalized profiles $\mathrm{h}(\mathrm{z})=\rho(\mathrm{z}) / \rho-1$, for different bulk concentrations $\rho$, are shown in Figure 9. The wallpolymer potentials $\phi(z ; \rho)$ were obtained using the wall$\mathrm{OZ}-\mathrm{HNC}$ inversion procedure and are plotted in Figure 10. The wall is essentially still hard but is now softened by an additional exponentially decaying repulsion. The density dependence is more pronounced than in the case of the effective potentials for bulk polymer solutions.

The potential for a polymer coil interacting with a colloidal HS of diameter $\sigma$ can be found from the density profile $\rho(r)$ around the sphere. Here $r$ is the distance from the center of the colloidal HS. The inversion procedure is very similar to that of the wall-polymer potential geometry. For a binary mixture of two components labeled $c$ and $p$, in which colloidal component $c$ is infinitely dilute $\left(\rho_{c} \rightarrow 0\right)$, the binary $\mathrm{OZ}$ equations decouple and reduce to

$$
h_{c p}(r)=c_{c p}(r)+\rho \int d r^{\prime} h_{c p}\left(\mathbf{r}^{\prime}\right) c_{b}\left(\left|\mathbf{r}-\mathbf{r}^{\prime}\right|\right)
$$

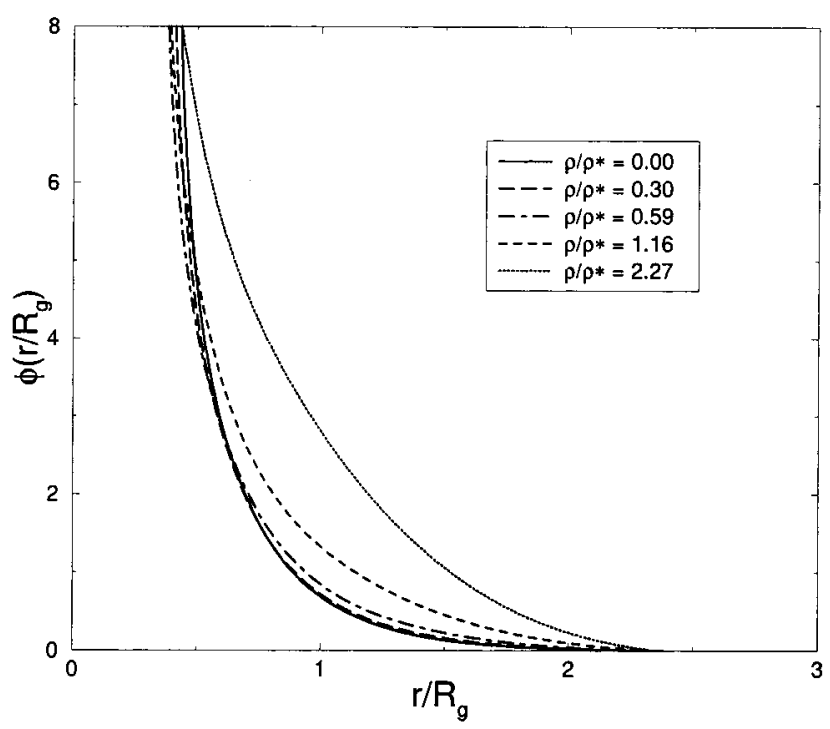

Figure 10. Wall-polymer potential $\phi(z ; \rho)$ as obtained from the inversion of density profile $\rho(z)$.

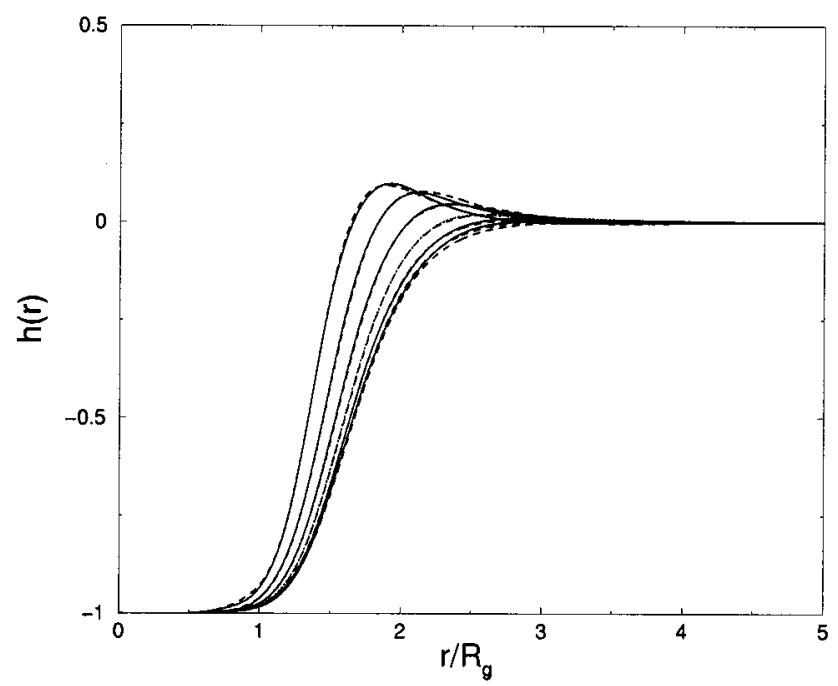

Figure 11. Polymer density profile $\mathrm{h}(\mathrm{r})=\rho(\mathrm{r}) / \rho$ around a colloidal HS of diameter $\sigma=2 \mathrm{R}_{\mathrm{g}}$. From left to right the curves correspond to a bulk polymer density $\rho / \rho^{*}=2.18,1.09,0.54$, $0.27,0.14$, and 0 . The solid lines represent the SAW simulation results, whereas the dashed lines correspond to the $h(r)$ that results from an optimized fit to the effective potential $\phi(r ; \rho)$.

where $h_{c p}(r)=\rho(r) / \rho-1$, and $c_{b}(r)$ is again the bulk polymer direct correlation function which is determined independently. The above equation can be combined with the HNC closure to yield

$$
\phi(r ; \rho)=-\ln (\rho(r) / \rho)+\rho \int d \mathbf{r}^{\prime} h_{c p}\left(r^{\prime}\right) c_{b}\left(\left|\mathbf{r}-\mathbf{r}^{\prime}\right|\right)
$$

which can be solved in one step by Fourier transformation. Figure 11 shows the polymer density profiles $h_{c p}(r)$ around a single colloidal HS particle with a diameter of $\sigma=2 \mathrm{R}_{\mathrm{g}}$. The effective potentials are plotted in Figure 12. The interaction appears to be somewhat softer than for the planar wall case, at least in the sense that it is still finite at distances that correspond to the CM being "inside" the hard colloidal particle. This happens more readily for smaller size ratios $\sigma /\left(2 \mathrm{R}_{\mathrm{g}}\right)$ because the polymers can deform around the colloids. Note that this penetration into the colloidal HS will not occur in the mid- or end-point representations.

3.4. Connection to Scaling Theory Approaches. Polymers in the semidilute regime have traditionally 


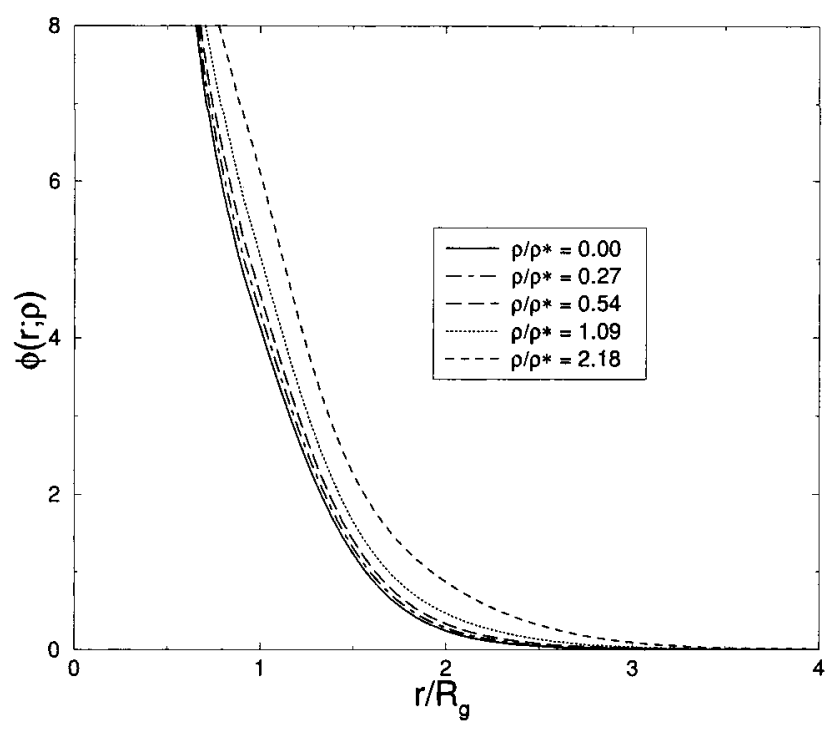

Figure 12. Colloid-polymer effective potential $\phi(r)$ for a colloidal diameter of $\sigma=2 \mathrm{R}_{\mathrm{g}}$.

been very successfully understood with scaling theories. ${ }^{21}$ There the fundamental unit is no longer the radius of gyration $\mathrm{R}_{\mathrm{g}}$ at zero density, but the blob size $\xi(\rho), 21,22$ which appears both in the EOS, where $\Pi_{b} \sim$ $\xi(\rho)^{-3}$, and in the relative adsorption $\Gamma / \rho \sim \xi(\rho)$. For the homogeneous polymer solutions, it is not clear from either the $g(r)$ or from the effective potentials where the blob size comes in. The reason the effective potentials correctly reproduce the EOS is because they correctly reproduce the pair correlations, which, through the compressibility theorem, ${ }^{29}$ give the correct thermodynamics. This bl ob size only appears in the potentials if one attempts to parametrize them for $\rho / \rho^{*}>2$ (roughly speaking, the density at which the scaling sets in), since the integral over $r^{2} v(r)$ should scale as $\rho^{-2} \xi(\rho)^{-3}$. Since we only parametrize the potentials for lower densities, this scaling does not apply to the functional form we use.

F or polymers near a wall the width of density profile of the monomers scales with $\xi(\rho)$ in the semidilute regime. ${ }^{21} \mathrm{~A}$ similar scaling is expected to hold for the width of the CM density profile. So even though $\xi(\rho)$ is not immediately evident in the effective wall-polymer potentials, it is present in the induced density profiles. Nevertheless, many questions as to the direct relationship between the scaling theories, and our soft-colloid approach remains to be worked out. Partially for that reason, some care must be taken when applying the softcolloid picture deep in the semidilute regime.

The semidilute regime scaling theories are not normally valid in the dilute regime, where we expect our soft-colloid approach to be robust.

\section{Fitting Effective Pair Potentials}

4.1. CM-CM Potentials for Bulk Polymer Solutions. When applying effective potentials in coarsegrained simulations or in theoretical analysis, it is convenient to have an explicit expression or parametrization of the potentials. In ref 16 we fitted the effective pair potentials we obtained by inversion of the $g(r)$ to a single Gaussian and described the remainder by means of a spline fit. However, for practical use in simulation or for a theoretical analysis, one would like to use an analytic expression without the complication of a multinode spline fit. Moreover, it would be very useful to be able to explicitly model the density dependence of the potentials as well. Since we are dealing with an approximately Gaussian form, the simplest analytical expression is a sum of Gaussians with density-dependent coefficients:

$$
\mathrm{v}_{\mathrm{f}}(\mathrm{r} ; \tilde{\rho})=\sum_{\mathrm{i}=0}^{\mathrm{n}} \mathrm{a}_{\mathrm{i}}(\tilde{\rho}) \mathrm{e}^{-\left(\mathrm{r} / \mathrm{b}_{\mathrm{i}}(\tilde{\rho})\right)^{2}}
$$

where we introduced $\tilde{\rho} \equiv \rho / \rho^{*}$ for clarity. Here, the Gaussians are centered at $r=0$, because the maximum of the potential is located at the origin. The density dependence comes in through the coefficients $a_{i}(\tilde{\rho})$ and $\mathrm{b}_{\mathrm{i}}(\tilde{\rho})$. I $\mathrm{n}$ the first instance, we take the coefficients to be linear in density, i.e., $\mathrm{a}_{\mathrm{i}}(\tilde{\rho})=\mathrm{a}_{\mathrm{i} 0}+\mathrm{a}_{\mathrm{i} 1} \tilde{\rho}$ and $\mathrm{b}_{\mathrm{i}}(\tilde{\rho})=\mathrm{b}_{\mathrm{i} 0}+$ $\mathrm{b}_{\mathrm{i} 1} \tilde{\rho}$.

Note that an expression of this form cannot describe the slightly negative tails in the potential that were found in ref 16. However, as was argued in the previous section, the structure is not very sensitive to small changes in $\mathrm{v}(r ; \tilde{\rho})$. A more important aspect of the fitting procedure is to make sure that the fitted potentials lead to the correct EOS. To do this, we make use of the accurate mean-fiel d approximation in eq 1. Because this is such a good approximation, keeping the $\mathrm{k}=0 \mathrm{~F}$ ourier component $\hat{v}(0 ; \tilde{\rho})=4 \pi \int r^{2} v(r ; \tilde{\rho})$ for the fitted potential $\mathrm{v}_{\mathrm{f}}(\mathrm{r} ; \tilde{\rho})$ equal to that of the original potential $\mathrm{v}(\mathrm{r} ; \tilde{\rho})$ ensures that the pressures will also be (almost) equal. A fitting procedure should therefore include the constraint

$$
\hat{\mathrm{v}}(0 ; \tilde{\rho})=\hat{\mathrm{v}}_{\mathrm{f}}(0 ; \tilde{\rho})=\pi^{3 / 2} \sum_{\mathrm{i}=0}^{\mathrm{n}} \mathrm{a}_{\mathrm{i}}(\tilde{\rho})\left(\mathrm{b}_{\mathrm{i}}(\tilde{\rho})\right)^{3}
$$

which results in one of the coefficients being fixed by the constraint. The $\hat{v}(0 ; \tilde{\rho})$ itself is a smooth function of the bulk density $\tilde{\rho}$; we fit it to a cubic polynomial .

The inverted potentials $v(r, \tilde{\rho})$ were fitted to eq 5 by applying a standard least-squares nonlinear fitting procedure, ${ }^{38}$ including the above constraint. To obtain a good fit of all the potentials for $\rho / \rho^{*}<2$, we needed at least three Gaussian terms, particularly to correctly describe $v_{f}(r=0 ; \tilde{\rho})$. For higher densities, the linear density dependence in eq 5 broke down. This can partly be understood from the fact that the potential at $r=0$ increases almost linearly for $\rho / \rho^{*}<2$ but decreases for higher concentration (see inset of Figure 1). Clearly, a linear fit in the density cannot cope with this behavior. Higher-order polynomials for the coefficients were not very successful either. A better option would be to fit the potentials for high densities independently and then ensure continuity at the crossover.

After using the nonlinear fitting procedure to directly fit the potentials, the best fit coefficients in eq 5 can be further fine-tuned by minimizing the difference between the radial distribution function $g(r)$ of the SAW polymers and the $g_{f}(r)$ generated from the fitted potential $\mathrm{v}_{\mathrm{f}}(\mathrm{r})$. This minimization procedure works as follows. For a certain density $\tilde{\rho}=\rho / \rho^{*}$, we first calculated $g_{f}(r)$ for $\mathrm{v}_{\mathrm{f}}(\mathrm{r} ; \tilde{\rho})$ with the best fit coefficients using the HNC approximation. We then compare this $g_{f}(r)$ to the original radial distribution function $g_{s A w}(r)$, measured in the explicit SAW simulation at the same density, by determining the least-squares difference: $\Delta(\tilde{\rho})=$ $\Delta\left(g_{f}(r)-g_{s A w}(r)\right)^{2} r^{2} d r$. We performed this calculation for every density $\tilde{\rho}_{\text {i }}$ we have SAW data for, summing 
Table 1. Best Fit Coefficients for v(r;ĩ) As Defined in Eq 5 for $L=500$ Polymers

\begin{tabular}{ccccc}
\hline $\mathrm{i}$ & $\mathrm{a}_{\mathrm{i} 0}$ & $\mathrm{a}_{\mathrm{i} 1}$ & \multicolumn{1}{c}{$\mathrm{b}_{\mathrm{i} 0}$} & $\mathrm{~b}_{\mathrm{i} 1}$ \\
\hline 1 & 1.47409 & -0.07689 & 0.981368 & -0.056808 \\
2 & -0.232096 & 0.031321 & 0.42123 & -0.026278 \\
3 & 0.638974 & 0.24193 & &
\end{tabular}

a Coefficients for $b_{3}(\tilde{\rho})$ are fixed by the constraint eq 7. The $\hat{v}(0 ; \tilde{\rho})$ in eq 7 is approximated by the cubic polynomial $\hat{v}(0 ; \tilde{\rho}) / 4 \pi=1.2902$ $+0.28132 \tilde{\rho}+0.136761 \tilde{\rho}^{2}-0.040892 \tilde{\rho}^{3}$

Table 2. Best Fit Coefficients for $v(r ; \tilde{\rho})$ As Defined in Eq 5 for $L=2000$ Polymers ${ }^{a}$

\begin{tabular}{rrrcc}
\hline $\mathrm{i}$ & \multicolumn{1}{c}{$\mathrm{a}_{\mathrm{i} 0}$} & \multicolumn{1}{c}{$\mathrm{a}_{\mathrm{i} 1}$} & $\mathrm{~b}_{\mathrm{i} 0}$ & $\mathrm{~b}_{\mathrm{i} 1}$ \\
\hline 1 & 1.41808 & -0.081969 & 0.979493 & -0.057796 \\
2 & -0.224377 & 0.030647 & 0.440907 & -0.024327 \\
3 & 0.630219 & 0.211378 & &
\end{tabular}

a Coefficients for $b_{3}(\tilde{\rho})$ are fixed by the constraint eq 7. The $\hat{v}(0 ; \tilde{\rho})$ in eq 7 is approximated by the cubic polynomial $\hat{v}(0 ; \tilde{\rho}) / 4 \pi=1.245$ $+0.3564275 \tilde{\rho}-0.02443297 \tilde{\rho}^{2}+0.0018028 \tilde{\rho}^{3}$

up to $\Delta_{\text {total }}=\sum_{\mathrm{i}} \Delta\left(\tilde{\rho}_{\mathrm{i}}\right)$. Subsequently, we changed one of the coefficients by a small amount and determined the difference $\Delta_{\text {total }}$ again. If the difference between the fitted $g_{f}(r)$ and the SAW data gsaw becomes smaller, the new coefficient was accepted; otherwise, the old coefficient was restored. These steps were repeated until the difference reached a minimum value, and changes in the coefficients were no longer accepted. The final coefficients only represent a local minimum, so this Monte Carlo type minimization procedure cannot be applied without a good initial estimate for the fit coefficients.. On the contrary, it depends strongly on the quality of the original nonlinear fit described above. Note that by using this optimization we skip one step, namely the inversion of the original $g(r)$. If a small error was made in the inversion, for example by our assumption that $v(r)=0$ for $r>r_{\text {cutoff, }}$ it should be corrected during the optimization. However, one must keep in mind that the minimization procedure only finds a local minimum. Larger errors may not be corrected.

We applied the above fitting procedure to the bulk polymer results for $L=500$ and $L=2000$. The coefficients are given in Table 1 and Table 2, respectively. One of the coefficients, $b_{3}(\tilde{\rho})$, is fixed by the constraint of eq 6:

$$
\left(b_{3}(\tilde{\rho})\right)^{3}=a_{3}(\tilde{\rho})^{-1}\left[\pi^{-3 / 2} \hat{v}(0 ; \tilde{\rho})-\sum_{i=0}^{2} a_{i}(\tilde{\rho})\left(b_{i}(\tilde{\rho})\right)^{3}\right]
$$

Note that this implies that, in contrast to the other coefficients, $b_{3}(\tilde{\rho})$ is no longer linear in density. The best fit coefficients for both lengths are not far from each other, again indicating that the results are close to the scaling limit.

To illustrate the importance of explicitly ind uding the constraint of eq 6, we applied the same nonlinear fitting procedure, including the Monte Carlo optimization, but without the constraint. In Figure 13 we compare $\hat{v}(0 ; \tilde{\rho})$, which is a good measure for the EOS, for the constrained and the unconstrained case. Clearly, including the constraint is essential for reproducing the correct thermodynamic behavior. At this point we would like to return to a statement made in an earlier paper, ${ }^{16}$ where we emphasized the importance of the very small negative tails we found from our inversion procedure. Because the tails are so small, their effect on the radial distribution functions is almost imperceptible. It is only
Table 3. Best Fit Coefficients for $\phi(z ; \tilde{\rho})$ As Defined in Eq 8 for $L=500$ Polymers Next to a Wall

\begin{tabular}{cccc}
\hline $\mathrm{i}$ & $\mathrm{a}_{\mathrm{i} 0}$ & $\mathrm{a}_{\mathrm{i} 1}$ & $\mathrm{a}_{\mathrm{i} 2}$ \\
\hline 0 & 62.7242 & 56.4595 & -29.92825 \\
1 & -6.40938 & -3.88795 & 2.044202 \\
2 & 2.50812 & 5.156190 & -2.13356 \\
3 & -0.69042 & -1.55191 & 0.59725
\end{tabular}

when they are multiplied by $r^{2}$ that they become important for the EOS. Our current fitting procedure ignores the negative tails, but because we force the sum rule on $r^{2} v(r)$, the thermodynamics are still correctly reproduced. Therefore, our previous statements on the importance of the tails should be tempered. It is actually the sum rule on the pair potential which is critical to achieving the correct thermodynamics.

4.2. Colloid-Polymer Potentials. A similar fitting procedure as in the previous section can be applied to the effective wall-polymer potentials. In ref 16 we showed that the effective wall-polymer potential $\phi(z, \tilde{\rho})$ can be fitted reasonably by the cubic exponential function

$$
\phi_{\mathrm{f}}(\mathrm{z}, \tilde{\rho})=\mathrm{a}_{0}(\tilde{\rho}) \exp \left[\mathrm{a}_{1}(\tilde{\rho}) \mathrm{z}+\mathrm{a}_{2}(\tilde{\rho}) \mathrm{z}^{2}+\mathrm{a}_{3}(\tilde{\rho}) \mathrm{z}^{3}\right]
$$

As before, the density dependence can be introduced through the coefficients $a_{i}(\tilde{\rho})=a_{i 0}+a_{i 1} \tilde{\rho}+a_{i 2} \tilde{\rho}^{2}$. Here we chose a quadratic density dependence, because a linear expression yielded a poor fit. We fitted the potentials $\phi(z ; \tilde{\rho})$ using the nonlinear fit method of the previous section but without any constraint. Subse quently, the coefficients were optimized using the minimization procedure mentioned above. The optimized best fit coefficients are given in Table 3, and the $\mathrm{h}_{\mathrm{f}}(\mathrm{z})$ generated by these fitted potentials are shown in Figure 9. The fit seems to be reliable for $\rho<\rho^{*}$ but, unfortunately, begins to break down in the semidilute regime. For the highest densities, the reproduced $h_{f}(z)$ shows more structure than the density profile obtained from the SAW simulations. Clearly, eq 8 cannot completely capture all the wall-polymer effects in the semi dilute regime. The functional form of eq 8 is rather ad hoc, and the fit would probably be better if one had access to a more accurate analytical expression based on physical arguments.

Similarly to the bulk polymer case, one needs to compare surface thermodynamic properties to ensure the quality of the fit. We focus on the polymer adsorption at the wall, $\Gamma$, defined as the partial derivative of the excess grand potential $\Omega^{\mathrm{ex}}$ per unit area with respect to the chemical potential $\mu$,

$$
\Gamma=-\frac{\partial\left(\Omega^{\mathrm{ex}} / \mathrm{A}\right)}{\partial \mu}=\rho \int_{0}^{\infty} \mathrm{h}(\mathrm{z}) \mathrm{dz}
$$

where $A$ is the surface area. In Figure 14 we show that the adsorption $\Gamma$ is indeed well represented by the fits. So, even though for high densities the structure next to the wall is not well described by eq 8, the integral over $h(z)$ is still accurately represented. Since the adsorption $\Gamma$ is given by the integral over $h(z)$, instead of an integral weighted by $z^{2}$, the tails of the potentials are less important to the correct thermodynamics than in the case of the bulk polymer potentials. In fact, if the adsorption $\Gamma$ and the EOS are known as a function of the density, then the surface tension of the polymer solution can be calculated with the Gibbs adsorption equation. ${ }^{35}$ Therefore, since the fitted wall-polymer 


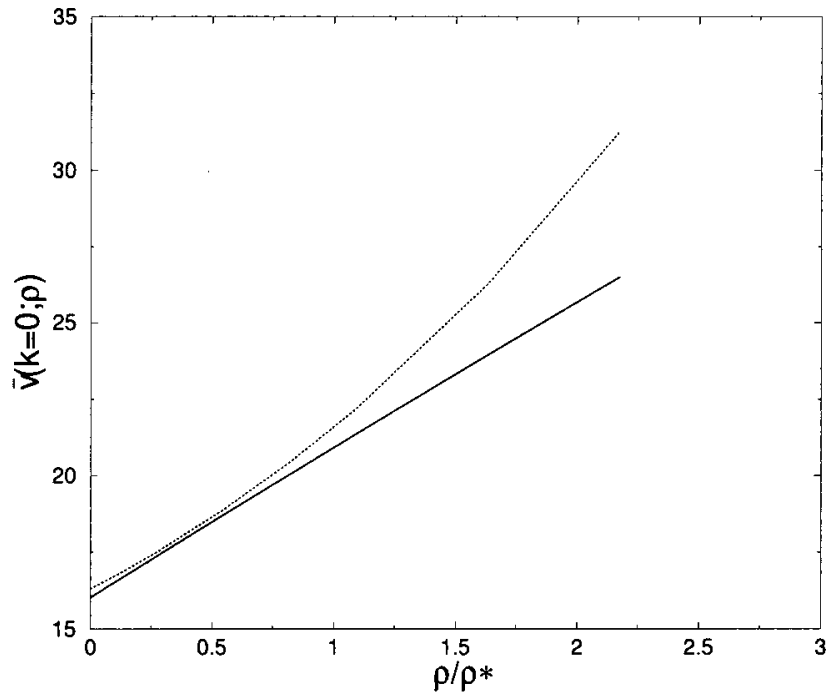

Figure 13. When the explicit constraint of eq 6 is not included, the value of $\hat{v}(k=0 ; \rho)=4 \pi \int r^{2} v(r ; \rho)$ (dotted line) begins to deviate significantly from the correct value (solid line). This will have an important effect on the EOS.

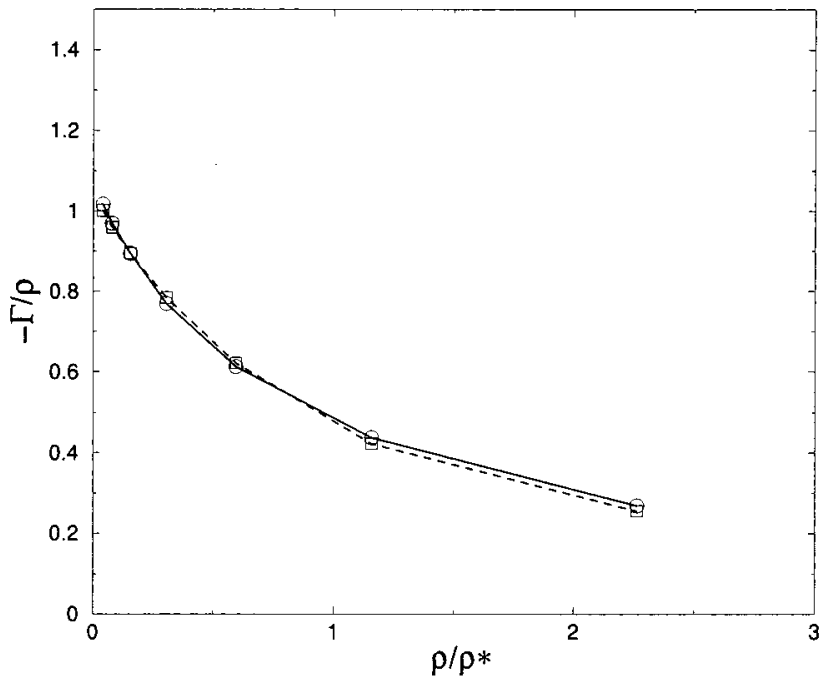

Figure 14. Relative adsorption $\Gamma / \rho$ (in units of $\mathrm{R}_{\mathrm{g}}{ }^{-2}$ ) given by direct SAW simulations of $L=500$ polymers next to wall (open circles) compares well to the adsorption cal culated from the fitted wall-polymer potentials (open squares).

potentials give the correct $\Gamma$ and the polymer-polymer potentials give the correct $\Pi_{b} / \rho$, when used together they should provide an accurate representation of surface tensions and related surface thermodynamic quantities.

In the case of a spherical particle, the fit to the sphere-polymer potential is more straightforward. Once again we can use a sum of Gaussians to estimate the potential

$$
\phi_{\mathrm{f}}(r)=\sum_{\mathrm{i}=0}^{\mathrm{n}} \mathrm{a}_{\mathrm{i}}(\tilde{\rho}) \mathrm{e}^{-\left(\left(\mathrm{r}-\mathrm{c}_{\mathrm{i}}(\tilde{\rho})\right) / \mathrm{b}_{\mathrm{i}}(\tilde{\rho})\right)^{2}}
$$

Here, because of the size difference between the colloidal particle and the polymer coil, we allow the Gaussians to be off center. In the nonlinear fit for the $\sigma=2 \mathrm{R}_{\mathrm{g}}$ data, we set $\mathrm{n}=2$ and $\mathrm{c}_{1}(\tilde{\rho})=0$ and assumed a linear density dependence of the coefficients. Subsequently, the coefficients were optimized using the minimization procedure. The results are given in Figure 11 and Table 4. The $h_{f}(r)$ reproduces the measured $h(r)$ quite well. To

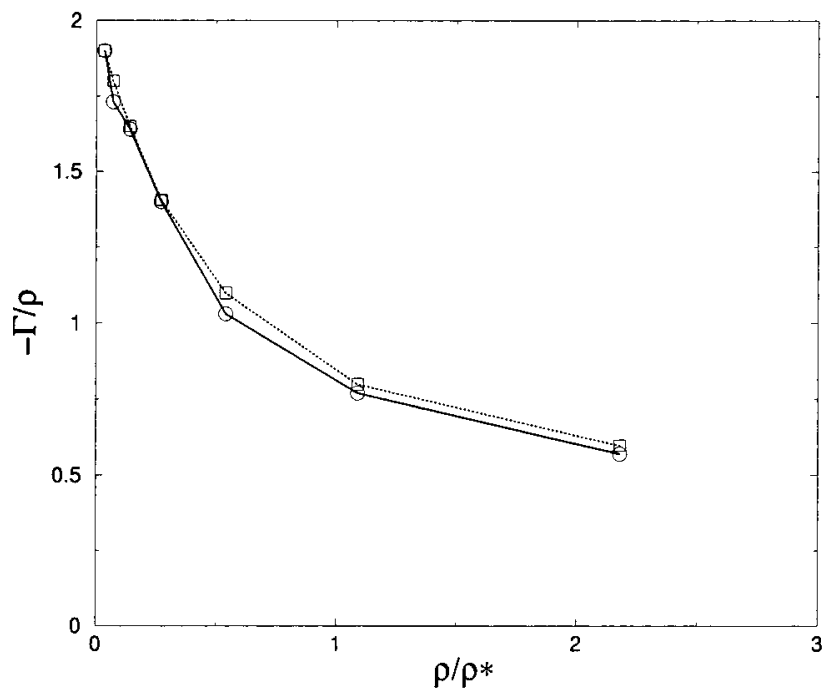

Figure 15. Relative adsorption $\Gamma / \rho$ (in units of $\mathrm{R}_{\mathrm{g}}{ }^{-2}$ ) given by direct SAW simulations of $L=500$ polymers around a colloidal sphere of diameter $\sigma=2 \mathrm{R}_{\mathrm{g}}$ (open circles) compared to the adsorption from the fitted colloid-polymer potentials (open squares). Note that the agreement is not as good as in the polymer wall case, because of the integration over $h(r) r^{2}$.

Table 4. Best Fit Coefficients for $\phi(r ; \tilde{\rho})$ As Defined in Eq 10 for $L=500$ Polymers around a Sphere of Diameter $\sigma=\mathbf{2}_{\mathbf{g}}$

\begin{tabular}{ccl}
\hline $\mathrm{i}$ & 0 & \multicolumn{1}{c}{1} \\
\hline $\mathrm{a}_{\mathrm{i} 0}$ & 5.5610 & 1.8477 \\
$\mathrm{a}_{\mathrm{i} 1}$ & -0.8042 & 1.4759 \\
$\mathrm{~b}_{\mathrm{i} 0}$ & 0.7751 & 1.2720 \\
$\mathrm{~b}_{\mathrm{i} 1}$ & -0.1151 & 0.1052 \\
$\mathrm{c}_{\mathrm{i} 0}$ & 0.4082 & 0.0 \\
$\mathrm{c}_{\mathrm{i} 1}$ & 0.14104 & 0.0
\end{tabular}

check the quality of the fit, we compared in Figure 15 the adsorption of the SAW polymers around the sphere with the optimized potentials. Here, in contrast to the planar wall case, we do have to integrate over $h(r) r^{2}$. We would therefore expect that the deviations are larger than for the wall. However, although the agreement is not as good as in the wall case, the adsorptions are still well represented, thus giving us confidence that the fits are adequate.

\section{Conclusion}

In this paper we have shown in detail how to use $\mathrm{OZ}$ techniques to derive effective potentials potentials for polymer solutions from direct simulations of SAW polymers. J ust as many subtleties were found in the original application of OZ inversion techniques to simple atomic and molecular fluids, so we find that great care must be taken to correctly invert and fit our effective potentials.

We found that $g(r)$ is not very sensitive to differences in $v(r)$, which is very similar to the situation for atomic and molecular fluids. ${ }^{29}$ This insensitivity places strong demands on the accuracy of the original $g(r)$ 's needed as input for our inversion procedures.

For the polymer-polymer potentials, it is crucial that the integral over $r^{2} v(r ; \rho)$ is correctly represented. Seemingly very small differences in the tails of $v(r)$, which in turn result in almost imperceptible changes in $g(r)$, can nevertheless result in large differences in the EOS. This principle should hold not only for linear polymers in the CM, end-point, or mid-point representations but 
also for low arm-number star polymers, 36 dendrimers, 39 and other mean-field fluids.

We also derived density-dependent wall-polymer and sphere-polymer potentials by directly inverting the oneparticle density profile $\rho(r)$ calculated by di rect simulations of $\mathrm{L}=500$ SAW polymers. Here, the important thermodynamic parameter is the adsorption $\Gamma$, which is quite well reproduced by our fitted potentials. Since our effective potentials provide a good representation of the EOS and of the adsorption, they should lead to an accurate representation of the surface tension and other related surface thermodynamic properties of polymer-colloid mixtures.

And finally, while one might think that most of the hard work is done once effective potentials have been inverted from direct simulations, fitting these potentials for their use in large-scale simulations of colloidpolymer mixtures is far from trivial. We showed how to use a numerical optimization procedure to ensure the accuracy of our fits. Because this optimization procedure skips the direct inversion step, it can remove residual errors in the inversions, guarantee that the fits conserve the right sum rules, and lead to the correct thermodynamics. We emphasize that these conclusions should hold for a much broader class of effective potentials than the ones we discussed here.

The ultimate goal of our research project ${ }^{15-17}$ is to model large-scale mixtures of polymers and colloids. The coarse-graining of the polymers from the "microscopic" SAW chains to single composite entities interacting via effective density-dependent pair potentials is a crucial step toward that goal. The next step will be to use the accurate fits derived in this paper to perform direct simulations of many spherical colloids interacting with many polymers.

Acknowledgment. A.A.L. acknowledges support from the Isaac Newton Trust, Cambridge. P.G.B. acknowledges support from the EPSRC under Grant GR/ M88839,

\section{References and Notes}

(1) Russel, W. B.; Saville, D. A.; Schowalter, W. R. Colloidal Dispersi ons; Cambridge University Press: Cambridge, 1989.

(2) Lekkerkerker, H. N. W.; Poon, W. C. K.; Pusey, P. N.; Stroobants, A.; Warren, P. B. Europhys. Lett. 1992, 20, 559564

(3) Meijer, E. J .; Frenkel, D. Phys. Rev. Lett. 1991 67, 11101113. Meijer, E. J .; Frenkel, D. J . Chem. Phys. 1994, 100, 6873-6887.

(4) Ilett, S. M.; Orrock, A.; Poon, W. C. K.; Pusey, P. N. Phys. Rev. E 1995, 51, 1344-1352.

(5) Verma, R.; Crocker, J . C.; Lubensky, T. C.; Yodh, A. G. Phys. Rev. Lett. 1998, 81, 4004-4007. Verma, R.; Crocker, J . C.; Lubensky, T. C.; Yodh, A. G. Macromol ecules 2000, 33, 177186.

(6) de Hoog, E. H. A.; Lekkerkerker, H. N. W.; Schulz, J .; Findenegg, G. H. J . Phys. Chem. B 1999, 103, 10657-10660.

(7) Poon, W. C. K.; Egelhaaf, S. U.; Stellbrink, J .; Allgaier, J .; Schofield, A. B.; Pusey, P. N. Philos. Trans. R. Soc. London, Ser. A 2001, 359, 897-908.

(8) Löwen, H.; Allahyarov, E.; Dzubiella, J .; von Ferber, C.; J usufi, A.; Likos, C. N.; Heni, M. Philos. Trans. R. Soc. London, Ser. A 2001, 359, 909-921.

(9) Yodh, A.; Lin, K.; Crocker, J . C.; Dinsmore, A. D.; Verma, R.; Kaplan, P. D. Philos. Trans. R. Soc. London, Ser. A 2001 359, 921-938.
(10) Louis, A. A. Philos. Trans. R. Soc. London, Ser. A 2001359 939-960.

(11) Evans, R.; Brader, J . M.; Roth, R.; Dijkstra, M.; Schmidt, M.; Löwen, H. Philos. Trans. R. Soc. London, Ser. A 2001 359, 961-976.

(12) Asakura, S.; Oosawa, F. J . Chem. Phys. 1954. 22, 1255-1256.

(13) Asakura, S.; Oosawa, F.J . Polym. Sci. 1958, 33, 183-184. Vrij, A. Pure Appl. Chem. 1976, 48, 471-483.

(14) Louis, A. A.; Finken, R.; Hansen, J . P. Europhys. Lett. 1999, 46, 741-747.

(15) Louis, A. A.; Bolhuis, P. G.; Hansen, J . P.; Meijer E. J . Phys. Rev. Lett. 2000, 85, 2522-2525.

(16) Bolhuis, P. G.; Louis, A. A.; Hansen, J. P.; Meijer, E. J . J Chem. Phys. 2001, 114, 4296-4311.

(17) Bolhuis, P. G.; Louis, A. A.; Hansen, J . P. Phys. Rev. E 2001 , 64, 021801.

(18) J ohnson, M. D.; Hutchinson, P.; March, N. H. Proc. R. Soc. London, Ser. A 1964, 282, 283-302.

(19) Reatto, L. Philos. Mag. A 1988, 58, 37-52. Reatto, L.; Levesque, D.; Weis, J.J. Phys. Rev. A 1986, 33, 3451-3465.

(20) Henderson, R. L. Phys. Lett. A 1974, 49, 197-198. Chayes, J . T.; Chayes, L. J . Stat. Phys. 1984, 36, 471-488.

(21) de Gennes, P. G. In Scaling Concepts in Polymer Physics; Cornell University Press: Ithaca, NY, 1979.

(22) Shäfer, L. In Excluded VolumeEffects in Polymer Solutions; Springer-Verlag: Berlin, 1999.

(23) Dautenhahn, J .; Hall, C. K. Macromol ecules 1994, 27, 5399 5412.

(24) Madras, N.; Sokal, A. D. J . Stat. Phys. 1988, 50, 109-186.

(25) Frenkel, D.; Smit, B. In Understanding Molecular Simulations; Academic Press: San Diego, 1995.

(26) Dijkstra, M.; Frenkel, D.; Hansen, J . P J . Chem. Phys. 1994 101, 3179-3189.

(27) Schmidt, M. Phys. Rev. E, in press.

(28) Louis, A. A.; Krakoviak, V.; Bolhuis, P. G.; Hansen, J . P., to be published.

(29) Hansen, J . P.; McDonald, I. R. In Theory of Simple Liquids, 2nd ed.; Academic Press: London, 1986.

(30) Louis, A. A.; Bolhuis, P. G.; Hansen, J . P. Phys. Rev. E 2000, 62, 7961-7972.

(31) Allen, M. P.; Tildesley, D. J . In Computer Simulation of Liquids; Oxford University Press: Oxford, 1987.

(32) Likos, C. N.; Lang, A.; Watzlawek, M.; Löwen, H. Phys. Rev. E 2001, 63, 031206.

(33) Dickman, R. J . Chem. Phys. 1987, 87, 2246-2248.

(34) Ohta, T.; Oono, Y. Phys. Lett. 1982, 89A, 460-464. See also: Schäfer, L. Macromolecules 1982, 15, 652-660.

(35) Mao, Y.; Bladon, P.; Lekkerkerker, H. N. W, Cates, M. E. Mol. Phys. 1997, 92, 151-159.

(36) Likos, C. N.; Löwen, H.; Watzlawek, M.; Abbas, B.; J ucknischke, O.; Allgaier, J .; Richter, D. Phys. Rev. Lett. 1998, 80, 4450-4453. Watzlawek, M.; Likos, C. N.; Löwen, H. Phys. Rev. Lett. 1999, 82, 5289-5292.

(37) Dzubiella, J .; J usufi, A.; Likos, C. N.; von Ferber, C.; Löwen, H.; Stellbrink, J .; Allgaier, J .; Richter, D.; Schofield, A. B.; Smith, P. A.; Poon, W. C. K.; Pusey, P. N. Phys. Rev. E 2000, 64, 010401.

(38) Press, W. H.; Teukolsky, S. A.; Vetterlin, W. T.; Flannery, B. P. In Numerical Recipes; Cambridge University Press: Cambridge, 1992

(39) Likos, C. N.; Schmidt, M.; Löwen, H.; Ballauff, M.; Pötschke, D.; Lindner, P. Macromolecules 2001, 34, 2914-2920.

(40) Note Added in Proof. We discovered a small error in our calculations of the radius of gyration. The value for radius of gyration mentioned in the text for $L=500$ is in reality $R_{0}$ $=16.83$ instead of $R_{g}=16.50$, and $R_{g}=38.23$ for $L=2000$ instead of $R_{g}=37.45$. This means that the distances in terms of $R_{\mathrm{g}}$ have to be rescaled by a factor of 0.9804 in case of $L=$ 500 and by a factor of 0.9796 in case of $L=2000$. All the reduced densities have to be rescaled by a factor of 1.0612 for $L=500$ and by 1.063 for $L=2000$. These small adjustments are only relevant when comparing to the theoretical results of the RG. They do not change, however, the validity of the approach nor any of the conclusions. Wethank Vincent Krakoviac for pointing out this error to us.

MA010888R 